\title{
Design of Health Service System Based on Cloud Platform
}

\author{
Yan Li, Yulei Huang, Qing Lin \\ School of Intelligence Science and Information Engineering \\ Xi'an Peihua University, Xi'an, China \\ 79132181@qq.com, 1397328804@qq.com, 530610232@qq.com
}

Keywords: Cloud platform; Health service; Data base; System design

\begin{abstract}
With the rapid development of modern society, people's quality of life is developing rapidly. At the same time, people's demand for health care system is also increasing. In recent years, the growing demand for the health system of the people has been far from meeting the needs of the common people. Based on the above reasons, this paper designs a health service system based on cloud platform, and carries out the requirement analysis, overall design and detailed design of the system. Through this system, blood pressure and blood glucose can be detected in real time, and the data can be viewed through the mobile phone. Finally, in order to realize the real-time storage of massive data after measurement, the system is based on the cloud platform to store the data.
\end{abstract}

\section{Introduction}

With the formal issuance of $4 \mathrm{G}$ license, China has entered the era of mobile Internet. Smart phone is not only used as our daily communication equipment, but also leaped to become a mobile platform for wireless connection to the Internet. Users can operate web browsing, file transfer and electronic transactions anytime, anywhere. In this mobile Internet age, mobile phones have completely changed the way people live. Through the mobile phone, it can also provide a new way of health management based on mobile phone platform - mobile health service. Mobile health service means that the traditional process of seeing a doctor is to register, see a doctor and treat in a hospital. Now these steps can be realized through mobile health service APP in mobile phone. Specifically, "mobile health service" is a scientific and efficient mobile health service system based on the mobile phone as the terminal, the "personal community" as the main body and the family community hospital government cooperation as the four in one using the 4G network and wireless access technology. The mobile platform to integrate the medical security resources, hospitals need health care human resources and medical devices resources through the integration of resources to play to the maximum extent the role of community medical staff of the newly formed "family", "community" and "cloud platform" by progressive and clear division of mobile health service system. In the mobile health service system, will need to use medical sensor connection, and collect the data fusion of the relevant medical data, finally the data is sent to the mobile phone terminal through the wireless network, the preliminary analysis and processing of the data generated a healthy curve and a health report, by accessing the mobile medical system can see at any time your health. The medical and health service system is in urgent need of transformation. People are also beginning to focus on the status of mobile health. It is urgent to improve and utilize the technology to solve the current situation of the medical and health system in the country.

The mobile medical system mentioned in this article refers to the installation of the APP application of the system in the mobile terminal. Mobile medical treatment, instead of people to the hospital and the treatment of the model, but through the App application on the mobile phone, anytime and anywhere to see their health. Through the detection equipment data measured by the database of mobile phone upload to the cloud platform in the cloud platform can be detected in their data sharing platform, the doctor can make a suggestion or problem is put forward on the platform for consultation, this way is applicable to some chronic diseases[1]. Mobile medical technology has achieved the role of saving money, time saving and labor saving through mobile communication technology, and has improved the quality of medical treatment, transforming the "treatment oriented" in the original medical mode into "prevention oriented". 


\section{System Requirement Analysis}

The system collects the health data of the human body through the equipment, including blood sugar data, blood pressure data and so on. After collecting the data, data transmission is carried out by SCDMA/GSM, and the data is finally displayed in the intelligent mobile terminal. The system uses the cloud platform[2], the measurement of large amounts of data generated in the process of storage, and in the process of processing the data buffer storage can realize the functions such as data and stored in the cloud platform data can be checked through the mobile phone, the data as the effective data reference.

\section{System Overall Design}

The health service system based on cloud platform mainly includes three parts: mobile health care system acquisition terminal[3], cloud platform for mobile medical health system, mobile phone health system based on Android mobile terminal. Cloud database platform acquisition terminal of mobile health care system will be measured data uploaded to the system, the database system of mobile health care system will mobile data transfer health care system to the mobile phone terminal, finally realize the user through the mobile phone terminal to access the data. The overall structure of the mobile medical health system is shown in Fig.1. in this article.

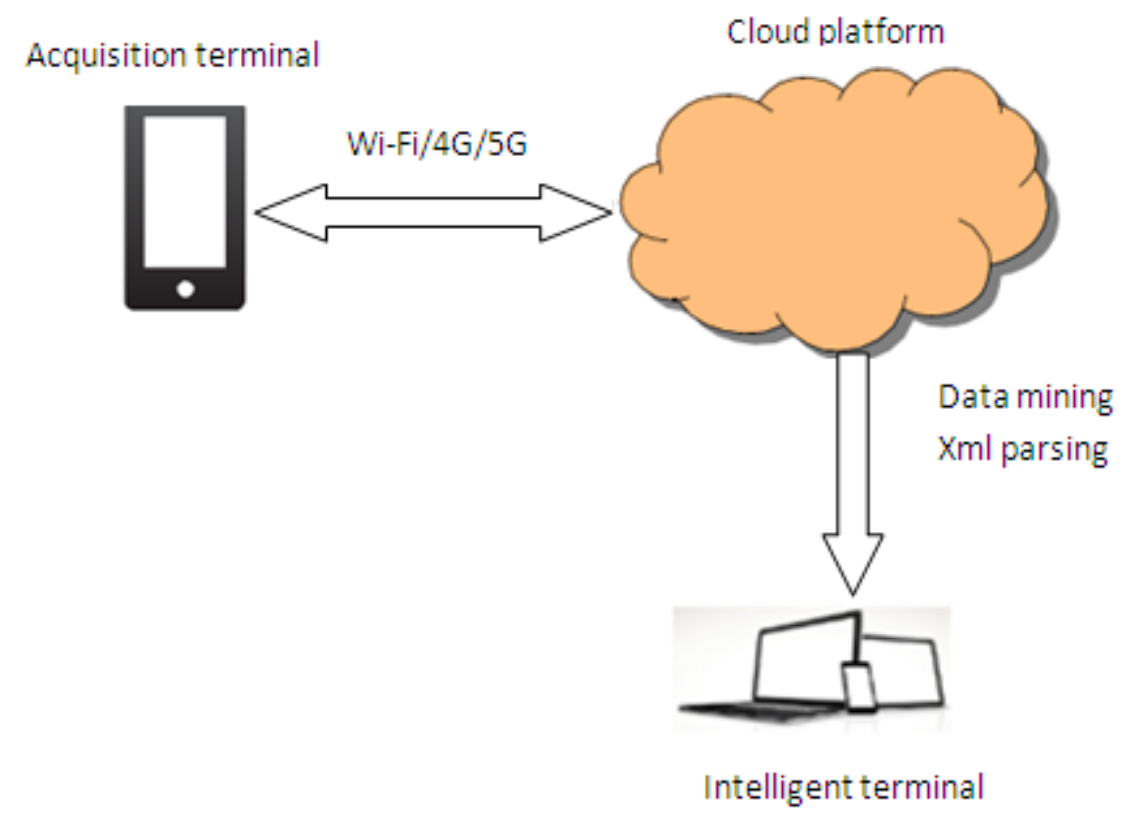

Figure 1. Overall structure of the system

\section{Detailed Design of the System}

\subsection{Design of Acquisition Terminal System}

The data collection of this system is composed of two parts: the blood sugar equipment and the blood pressure equipment. The functional block diagram of the system acquisition terminal is shown in Fig. 2. The system can store multiuser blood glucose data and blood pressure data. The acquisition terminal is interconnected with medical equipment through blue tooth and other ports to realize the collection of blood glucose data and blood pressure data. 


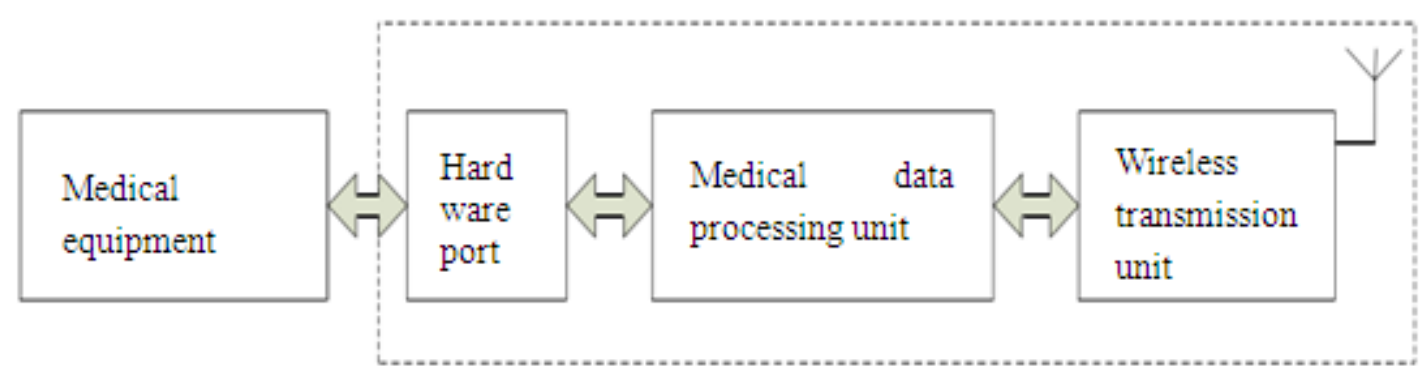

Figure 2. Design of acquisition terminal system

\subsection{Design of Data Acquisition Module}

The data acquisition module mainly consists of two parts of blood sugar and blood pressure. At present, the measurement of blood sugar and blood pressure is not timely, the data is easy to lose, and the whole measurement process is prone to leak detection, error test and so on. Based on this, this paper designed the data of blood glucose measurement and blood pressure measurement data acquisition module[4].

1. Blood sugar is an important indicator of the human body

Hypoglycemia can lead to slow response, the body tired; as everyone knows, the high blood sugar caused by diabetes, long-term diabetes control can cause pathological changes of various organs of the body, cause a series of complications, in daily life, regardless of age or time of blood glucose monitoring population has been suffering from the disease is necessary. At present, the medical situation is mainly monitored by the patients themselves in hospitals or places where they can be measured. The whole process is time-consuming and energy consuming, and there are still many problems. The main problem is that data cannot be stored for long. This paper is designed from this point to realize the automatic uploading of measurement data by a measuring instrument, so as to reduce the inaccuracy of data and preserve historical data. The blood glucose module is designed in this paper can be directly connected by blood glucose meter, blood glucose meter measurement data can be uploaded to the cloud platform, cloud platform has its own health records, we realize these historical data is automatically saved, at the same time can be generated according to the number of historical curve, blood glucose monitoring long-term users.

The glycemic instrument mentioned in this article is a universal blood glucose test device, and the communication port is usually a serial port.

The blood sugar module is divided into the following steps according to the working process:

(1) the single chip computer is needed at present, and the first step on the single chip computer;

(2) the single chip computer after electricity, no work, users need to wake up the single chip computer to make it work, so as to obtain blood glucose data;

(3) the single chip computer uploads the blood sugar data to the cloud platform through the wireless transmission module.

2. The Design of the Sphygmomanometer Module

Blood pressure is a common indicator of the health of the human body. After the blood pressure measurement is compared with the standard data, the blood pressure of the user can be mastered and the problem can be found in time. Therefore, it is particularly important to monitor blood pressure in daily life.

The content mentioned in the paper is that the user's blood pressure measurement is not timely, and data is easy to lose. Therefore, based on the above problems, we designed the sphygmomanometer module, and the sphygmomanometer module first obtained the blood pressure data of the human body through the blood pressure measuring instrument, and the measured data were saved in time. We use MCU to communicate with GPRS, and finally transmit data to cloud platform through data communication module. Users can access these data through mobile terminals. 


\subsection{Design of Cloud Platform Database}

This system has the support of large database, which provides the function of storing massive data. Through Paas, it provides many users with high security and reliability of database service. The database framework used in this article includes the presentation layer (PL) [5], the business logic layer (BLL), and the database access layer (DAL), as shown in Fig.3.

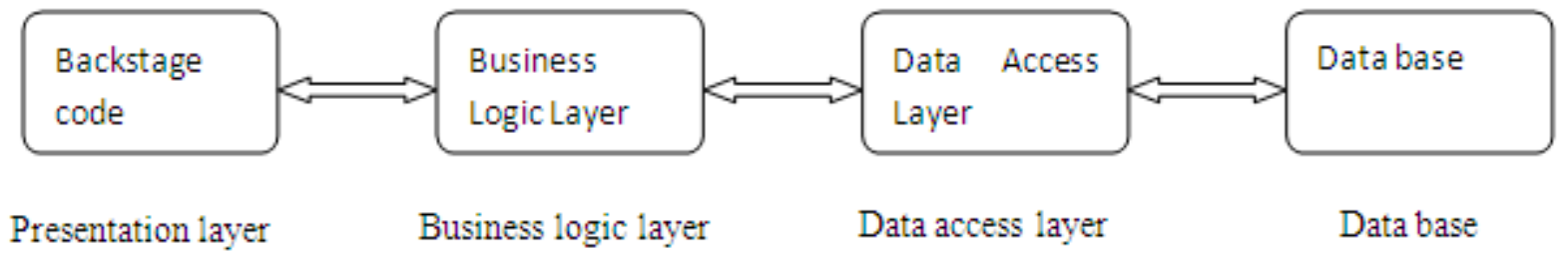

Figure 3. Function process of database system

\subsection{Design of Mobile Intelligent Terminal}

At present, with the rapid development of science and technology, smart phone is becoming more and more widely used, and its functions are becoming more and more powerful. Because the Android system's mobile phone is easy to operate, convenient and flexible and so on, it has been favored by many users. In this design, the top mobile medical service system cloud platform based on application layer, Android platform is the design of a mobile medical intelligent terminal based on client, through the system, users can use mobile phone to complete view real-time health monitoring data.

According to the module partition, the system is divided into seven parts: user registration module, login module, data receiving module, judgment data module, data download module, data statistics module and user information management module.

\section{Conclusions}

This paper analyses the requirements of health service system, overall design, detailed design, put forward the overall framework of mobile health care system based on cloud platform, the framework including blood pressure, blood sugar collection terminal equipment, cloud platform database, storage of large capacity data for intelligent mobile phone with three parts, this paper carried out a detailed design on the above mentioned three parts. The full text is designed according to the actual situation. The train of thought is rigorous and hierarchical. According to the data transmission process needed in the medical health system, the overall conception and design is carried out, which is easy to understand and lay the foundation for further development of the system.

\section{References}

[1] J. Zhu. 2018. Research and implementation of SaaS cloud platform for community intelligent vending machines, Computer application research, 4:198-199.

[2] J. Yuan. 2018. Design and implementation of large data experimental cloud platform, information technology, 2:70-72.

[3] Q. Cheng. 2013. The current situation and trend of global mobile medical development, Shanghai informatization, 5:80-82.

[4] H. Xie. 2012. Unstructured data access for cloud storage, f computer application, 32(7):1924-1928.

[5] H. Ni. 2013. Research on data storage access mechanism based on Android system, computer technology and development, 6:90-93. 